\title{
The Cohort Study on Prediction of Incidence of All-Cause Mortality by Metabolic Syndrome
}

\author{
Zhixia Li ${ }^{1}$, Xinghua Yang ${ }^{2}$, Jun Yang ${ }^{1}$, Zhirong Yang ${ }^{1}$, Shengfeng Wang ${ }^{1}$, Feng Sun ${ }^{1 \oplus *}$, \\ Siyan Zhan ${ }^{1 \odot *}$ \\ 1 Department of Epidemiology and Biostatistics, School of Public Health, Peking University Health Science \\ Center, Beijing, China, 2 Department of Epidemiology and Health Statistics, School of Public Health, Capital \\ Medical University, Beijing, China \\ ه These authors contributed equally to this work. \\ * siyan-zhan@ @jmu.edu.cn (SYZ); sunfeng @ bjmu.edu.cn (FS)
}

\section{Abstract}

\section{Aim}

The aim was to evaluate the impact of metabolic syndrome (MS), MS individual components and 32 kinds of MS specific component combinations on all-cause mortality risk in a fixed cohort of MJ check-up population.

Citation: Li Z, Yang X, Yang J, Yang Z, Wang S, Sun F, et al. (2016) The Cohort Study on Prediction of Incidence of All-Cause Mortality by Metabolic Syndrome. PLOS ONE 11(5): e0154990. doi:10.1371/ journal.pone. 0154990

Editor: Ying-Mei Feng, Beijing Key Laboratory of Diabetes Prevention and Research, CHINA

Received: July 13, 2015

Accepted: April 23, 2016

Published: May 19, 2016

Copyright: $\odot 2016 \mathrm{Li}$ et al. This is an open access article distributed under the terms of the Creative Commons Attribution License, which permits unrestricted use, distribution, and reproduction in any medium, provided the original author and source are credited.

Data Availability Statement: All relevant data are within the paper and its Supporting Information files.

Funding: This study is funded by National Natural Science Foundation of China (81302508) and Research Fund for the Doctoral Program of Higher Education (20120001110015).

Competing Interests: The authors have declared that no competing interests exist.

\section{Methods}

We observed the events of death in a fixed cohort, where the population was composed of 45,542 individuals aged 35-74 who were examined at MJ Health check-up Center in 1997 as baseline examination, and were followed up to 2005. Median duration of follow-up was 7.44 years. MS was defined according to the National Cholesterol Educational Program (the revised NCEP-ATPIII for Asian in 2004), the prevalence of MS was standardized according to China's fifth census data. We constructed common Cox regression model, simultaneously adjusting the classic risk factors (such as age, sex, smoking, alcohol drinking, physical activity, family history, etc.) to examine the relationship between MS, MS individual components and 32 kinds of MS specific component combinations on the occurrence of death with the fixed cohort.

\section{Results}

The standardized prevalence of MS was $29.75 \%$ (male: $30.36 \%$, female: $29.51 \%$ ). There were 1,749 persons who died during the median 7.44-years follow-up, the mortality rate was 46 per 10,000 person years. The mortality rates were 71 and 35 per 10,000 person years for those with and without MS, respectively. After adjustment for age, sex and classical risk factors, compared with subjects without MS, the hazard ratio of all-cause mortality was 1.26 (95\% Cl: 1.14-1.40). The all-cause mortality were more highly significant than other combinations $(P<0.05)$ when the following combinations exist: "elevated blood pressure", "elevated fasting plasma glucose + low high-density lipoprotein cholesterol", 
"elevated blood pressure + elevated triglyceride + elevated fasting plasma glucose", "elevated fasting plasma glucose + low high-density lipoprotein cholesterol + elevated blood pressure + elevated triglyceride". After adjusting age, sex and classical risk factors, the HRs for those with 0 to 5 components were $1,1.22,1.25,1.33,1.66$, and 1.92, respectively. There was a significant dose-response relationship ( $P$ for liner trend $<0.001$ ) between the number of MS components and the risk of all-cause mortality in the overall fixed cohort sample.

\section{Conclusion}

In a large scale middle-aged Taiwan check-up population, MS may be associated with a much higher risk for all-cause mortality. These results may underline the fact that MS is a non-homogeneous syndrome and have a significant impact on detecting high-risk individuals suffering from metabolic disorders for preventing and controlling death.

\section{Introduction}

Metabolic syndrome (MS), which refers to a group of metabolic and vascular disorders that occur simultaneously, has been found in meta-analyses to increase the risk of all-cause mortality in the general population[1-3]. Compared with its individual components, the prognostic importance of MS has repeatedly been challenged and could not be addressed in the meta-analyses. Zambon's study showed that not all individual components of MS contributed to the increased risk of all-cause mortality [4].

Since previous studies investigating the relationship between MS and mortality were primarily conducted in Europe and North America, and it remains one of the least studied factors in China $[5,6]$. Whether and the degree to which different MS specific component combinations portend greater risk for all-cause mortality has rarely been investigated. The first objective of the present study was to explore the association of MS, MS individual components and 32 kinds of MS specific component combinations with all-cause mortality in a Taiwanese cohort. Secondly, we also tested the hypothesis whether consideration of different number of MS components as a risk continuum was a more valuable risk predictor than identifying MS. Thirdly, we examined which MS specific component combinations pose the greatest risk for all-cause mortality. Fourth, the objectives above were further explored after excluded subjects with essential hypertension (EH), type 2 diabetes mellitus (T2DM) and Chronic kidney diseases (CKD), who were at high risk for all-cause mortality[6-9].

\section{Material and Method}

\section{Study samples}

This is a retrospective cohort study and baseline data was collected from MJ Health Screening Centers (Taipei, Taoyuan, Taichung and Kaohsiung) in Taiwan between 1 January 1997 and 31 December 1997. Details of the MJ centers are described elsewhere [10-13]. MJ Health Screening Centers attracted paying participants from all over Taiwan because of its known quality services, operational efficiency, and key facilities that were easily accessible. Memberships to the programme was required, with discounts in examination fees offered for people with large-size family or related individuals and for regular members who came back for repeated examinations in subsequent years, and these incentives succeeded in attracting and 
sustaining a large number of customers. These centers provide a multidisciplinary team approach for their members, and members usually undergo health examination every 3-4 years, about $30 \%$ of them will receive a health examination every year. All subject data were recorded into a database using standardized data sheets. Demographic profiles of the members are similar to those of the general Taiwanese population. Deaths were ascertained by computer linkage to the national death registry using ID numbers. Every cause of death was certified by a registered medical doctor in Taiwan and recorded by a staff at the local health bureau.

Subjects aged 35-74 years and could be diagnosed as MS or Non-MS were recruited. Subjects were excluded if their baseline information were missing, including age, sex, smoking, alcohol drinking, physical activity, EH family history and T2DM family history.

\section{Ethics statement}

This study has been approved by Peking University Institutional Review Board, who made the following decision: this study has eliminated all identifiable personal information, not belonging to studies involving human beings, so we grant waivers of informed consent and ethical review to it. All or part of the data used in this research were authorized by, and received from MJ Health Research Foundation (Authorization Code: MJHRFB2014003C). Any interpretation or conclusion described in this paper does not represent the views of MJ Health Research Foundation.

\section{MS definitions}

In the present study, we used National Cholesterol Educational Program (the revised NCEP-ATPIII for Asian in 2004) [14] to define MS, which requires the presence of 3 or more of the following: (i) waist circumference (WC) $\geq 102 \mathrm{~cm}$ in men and $\geq 88 \mathrm{~cm}$ in women, (ii) hypertriglyceridemia (TG) $\geq 1.7 \mathrm{mmol} / \mathrm{l}$, (iii) low high-density lipoprotein cholesterol(HDL-C) $<1.04$ $\mathrm{mmol} / \mathrm{l}$ in men and $<1.3 \mathrm{mmol} / \mathrm{l}$ in women, (iv) blood pressure: (BP) hypertension with systolic $\geq 130 \mathrm{mmHg}$ or diastolic $\geq 85 \mathrm{mmHg}$, (v) fasting plasma glucose (FPG) $\geq 6.1 \mathrm{mmol} / \mathrm{l}$.

\section{Follow-up and endpoints}

Subjects were classified based on the presence or absence of MS, and were prospectively followed until 31 December 2005 or until death, if this event occurred earlier. All deaths that occurred from the study entry to 21 December 2005 were categorized as all-cause deaths.

\section{Statistical analysis}

The demographic characteristics of the study subjects were expressed as means \pm standard deviation for continuous variables or as percentages for categorical variables. Continuous variables were compared using a two-sided Student's t-test between the two groups classified by the presence or absence of MS. Categorical variables were compared using the chi-square test.

The prevalence of MS was standardized according to China's fifth census data. Distribution of the study subjects among different number of MS components was descripted. Mortality rate of MS individual components were calculated.

Log-Rank test was used to determine proportional hazard assumption. Hazard ratios (HR), and 95\% CI for all-cause mortality for MS individual components and 32 kinds of MS specific components combinations were analyzed with forced Cox proportional hazards regression models, with crude models (Model 1); with adjustment for age, sex (Model 2); with adjustment for age, sex and classical risk factors including education, smoking, alcohol drinking, physical 
activity, EH family history and T2DM family history (Model 3). These models were further used after excluded subjects with EH or T2DM or CKD.

A P-value $<0.05$ was considered statistically significant. All statistical calculations were performed using SPSS 20.0 for Windows.

\section{Results}

\section{Baseline characteristics}

A total of 45,542 members were recruited. Flow chart of subject selection was shown in Fig 1. The overall trend of age composition ratio of the cohort subjects was similar to that of the standard population[15]. (Table A in S1 File and Fig A in S2 File)

The standardized prevalence of MS was $29.75 \%$ (male: $30.36 \%$, female: $29.51 \%$ ). Statistically significant difference was found among different groups categorized by age (results not shown). (Table B in S1 File)

Of the 45,542 subjects included, 13,979 subjects (30.69\%) had MS by NCEP-ATP III criteria.The baseline characteristics of MS group and Non-MS group are shown in Table 1.

\section{Relationships between baseline MS individual components}

The relationships between baseline MS individual components were shown in Table C in S1 File. Nearly $60 \%$ subjects with central obesity had a low level of HDL-C or an elevated level of $\mathrm{BP}$ at the same time. 50.53\% subjects with elevated BP also had a low level of HDL-C. There

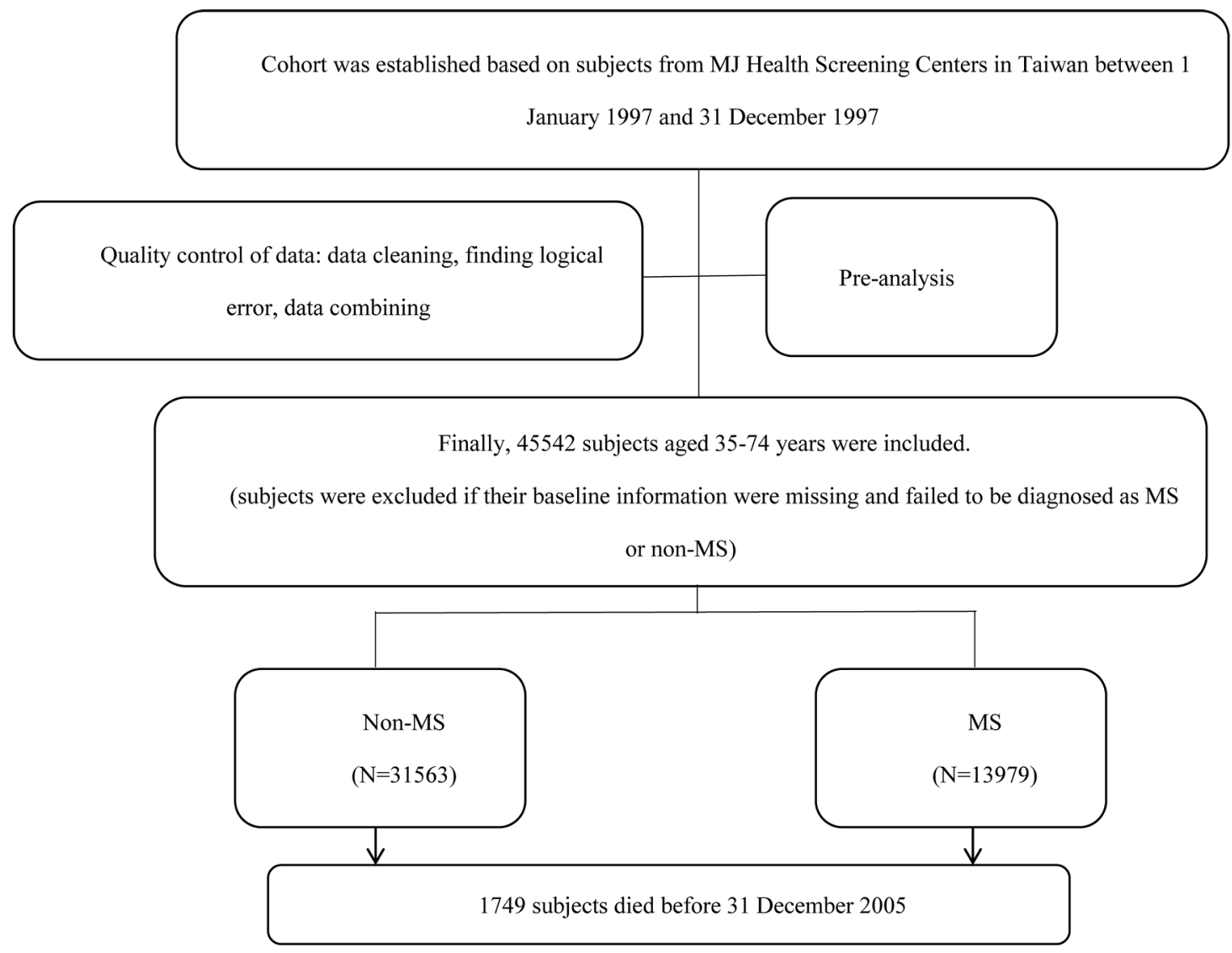

Fig 1. Flow chart of subject selection.

doi:10.1371/journal.pone.0154990.g001 
Table 1. Baseline Characteristics $(\mathrm{N}=45542)$.

\begin{tabular}{|c|c|c|c|}
\hline & Non-MS( $n=31563)$ & $M S(n=13979)$ & $\mathbf{P}$ \\
\hline Sex & & & 0.898 \\
\hline Male & $14638(46.38)$ & $6474(46.31)$ & \\
\hline Female & 16925 (53.62) & 7505 (53.69) & \\
\hline Age (years) & $48.25 \pm 10.3$ & $54.63 \pm 10.36$ & $<0.001$ \\
\hline Age gruop & & & $<0.001$ \\
\hline 35- & $14226(45.07)$ & 2952 (21.12) & \\
\hline $45-$ & $8125(25.74)$ & $3481(24.90)$ & \\
\hline $55-$ & $6480(20.53)$ & 4797 (34.32) & \\
\hline $65-74$ & $2732(8.66)$ & 2749 (19.67) & \\
\hline T2DM family history & 3405 (10.79) & $1687(12.07)$ & $<0.001$ \\
\hline EH family history & $5116(16.21)$ & $2608(18.66)$ & $<0.001$ \\
\hline Cerebrovascular history & $1101(3.49)$ & $516(3.69)$ & 0.280 \\
\hline Education (high school or above) & 8625 (27.33) & $2377(17.00)$ & $<0.001$ \\
\hline Marriage & & & $<0.001$ \\
\hline unmarried & $993(3.28)$ & $182(1.36)$ & \\
\hline married & 26797 (88.40) & $11540(86.37)$ & \\
\hline divorce & $864(2.85)$ & $212(1.59)$ & \\
\hline death of a spouse & $1661(5.48)$ & $1427(10.68)$ & \\
\hline physical activity & & & $<0.001$ \\
\hline little & 13695 (43.39) & $5892(42.15)$ & \\
\hline Occasionally & 6197 (19.63) & $2493(17.83)$ & \\
\hline regularly & $3252(10.30)$ & $1280(9.16)$ & \\
\hline Every day & $6076(19.25)$ & 3265 (23.36) & \\
\hline Smoking & & & $<0.001$ \\
\hline Yes & $6674(22.86)$ & $2798(21.71)$ & \\
\hline No, Has given up & $2050(7.02)$ & $1118(8.67)$ & \\
\hline Never & $20472(70.12)$ & 8975 (69.62) & \\
\hline Smoking frequency (one day) & & & $<0.001$ \\
\hline $1-10$ & 2856 (33.45) & $1192(30.96)$ & \\
\hline $10-20$ & 3615 (42.34) & $1551(40.29)$ & \\
\hline$\geq 20$ & $2067(24.21)$ & $1107(28.75)$ & \\
\hline alcohol drinking & & & $<0.001$ \\
\hline Yes & $6415(22.11)$ & $2855(22.43)$ & \\
\hline No, Has given up & $903(3.11)$ & $500(3.93)$ & \\
\hline Never & $21699(74.78)$ & $9373(73.64)$ & \\
\hline Height (cm) & $160.76 \pm 8.10$ & $160.11 \pm 8.65$ & $<0.001$ \\
\hline Weight (Kg) & $58.86 \pm 9.46$ & $66.78 \pm 11.00$ & $<0.001$ \\
\hline BMI $\left(\mathrm{Kg} / \mathrm{m}^{2}\right)$ & $22.72 \pm 2.82$ & $25.97 \pm 3.21$ & $<0.001$ \\
\hline BMI group & & & $<0.001$ \\
\hline$<18.5 \mathrm{Kg} / \mathrm{m}^{2}$ & $1768(5.60)$ & $68(0.49)$ & \\
\hline $18.5-24.9 \mathrm{Kg} / \mathrm{m}^{2}$ & $23714(75.1)$ & $5573(39.87)$ & \\
\hline $24.9-29.9 \mathrm{Kg} / \mathrm{m}^{2}$ & $5693(18.04)$ & 6940 (49.65) & \\
\hline $30-34.9 \mathrm{Kg} / \mathrm{m}^{2}$ & $360(1.14)$ & $1255(8.98)$ & \\
\hline $35-39.9 \mathrm{Kg} / \mathrm{m}^{2}$ & $21(0.07)$ & $122(0.87)$ & \\
\hline$\geq 40 \mathrm{Kg} / \mathrm{m}^{2}$ & $3(0.01)$ & $19(0.14)$ & \\
\hline WC (cm) & $77.71 \pm 8.40$ & $88.04 \pm 8.56$ & $<0.001$ \\
\hline Central OB* & $5133(16.26)$ & 10194 (72.92) & $<0.001$ \\
\hline
\end{tabular}


Table 1. (Continued)

\begin{tabular}{|c|c|c|c|}
\hline & Non-MS(n = 31563) & $\operatorname{MS}(n=13979)$ & $\mathbf{P}$ \\
\hline WHR & $0.83 \pm 0.07$ & $0.89 \pm 0.07$ & $<0.001$ \\
\hline Body fat (\%) & $24.85 \pm 6.93$ & $29.69 \pm 7.61$ & $<0.001$ \\
\hline Pulse (times) & $74.28 \pm 9.96$ & $77.07 \pm 11.16$ & $<0.001$ \\
\hline FPG (mg/dl) & $95.28 \pm 16.85$ & $112.89 \pm 36.86$ & $<0.001$ \\
\hline TC (mg/dl) & $197.52 \pm 36.21$ & $210.73 \pm 40.26$ & $<0.001$ \\
\hline TG (mg/dl) & $100.53 \pm 50.59$ & $178.61 \pm 91.95$ & $<0.001$ \\
\hline HDL-C (mg/dl) & $49.16 \pm 13.35$ & $39.88 \pm 11.66$ & $<0.001$ \\
\hline LDL-C (mg/dl) & $128.34 \pm 31.89$ & $135.84 \pm 36.00$ & $<0.001$ \\
\hline SBP (mmHg) & $118.67 \pm 18.86$ & $138.21 \pm 21.65$ & $<0.001$ \\
\hline DBP (mmHg) & $73.38 \pm 10.61$ & $81.45 \pm 11.83$ & $<0.001$ \\
\hline CRP (mg/dl) & $0.23 \pm 0.53$ & $0.32 \pm 0.73$ & $<0.001$ \\
\hline ALT (IU/L) & $24.5 \pm 28.54$ & $31.70 \pm 31.04$ & $<0.001$ \\
\hline AST(IU/L) & $23.74 \pm 19.05$ & $26.52 \pm 19.26$ & $<0.001$ \\
\hline $\operatorname{GFR}\left(\mathrm{mL} / \mathrm{min} / 1.73 \mathrm{~m}^{2}\right)$ & $79.84 \pm 14.45$ & $74.48 \pm 15.35$ & $<0.001$ \\
\hline BUN (mg/dl) & $14.97 \pm 4.25$ & $16.02 \pm 5.48$ & $<0.001$ \\
\hline CRE (mg/dl) & $0.96 \pm 0.28$ & $1.02 \pm 0.48$ & $<0.001$ \\
\hline UA (mg/dl) & $5.73 \pm 1.55$ & $6.44 \pm 1.64$ & $<0.001$ \\
\hline Working on the seat & $14314(48.87)$ & $6053(47.73)$ & $<0.001$ \\
\hline Often drinking milk & $5452(18.29)$ & $1750(13.38)$ & $<0.001$ \\
\hline Often eating fruit & $16883(53.49)$ & 6967 (49.84) & $<0.001$ \\
\hline Often drinking SSB & $2675(9.25)$ & $996(7.87)$ & $<0.001$ \\
\hline EH & $5433(17.21)$ & $8044(57.54)$ & $<0.001$ \\
\hline T2DM & $602(1.91)$ & 2057 (14.71) & $<0.001$ \\
\hline CKD & 3778 (11.97) & 3603 (25.77) & $<0.001$ \\
\hline
\end{tabular}

Data are mean \pm standard deviation or number (\%). BMI: body mass index; WC: waist circumference; OB: obesity; WHR: Waist-to-hip ratio; FPG: fasting plasma glucose; TC: Serum total cholesterol; TG: Triglyceride; HDL-C: high-density lipoprotein cholesterol; LDL-C: low density lipoprotein-cholesterol; SBP: systolic blood pressure; DBP: diastolic blood pressure; CRP: C-reaction protein; ALT: alanine transaminase; AST: Aspartate aminotransferase; GFR: glomerular filtration rate, GFR (mL/min per $1.73 \mathrm{m3})=186.3 \times($ Serum creatinine $)-1.154 \times$ Age- $0.203 \times(0.742$ if female); BUN: blood urea nitrogen; CRE: Serum creatinine; UA: uric acid; SSB: sugar-sweetened beverages; EH: essential hypertension; T2DM: type 2 diabetes mellitus; CKD: Chronic kidney diseases.

*: Central obesity was defined as waist circumference $\geq 90$ for male and $\geq 80$ for female.

doi:10.1371/journal.pone.0154990.t001

was $65.95 \%$ subjects with elevated TG had a low level of HDL-C. $43.35 \%$ subjects with low HDL-C had an elevated level of BP at the same time. There was $56.74 \%$ subjects with elevated FPG had an elevated level of BP, too.

Table 2 showed the distribution of the study subjects among different number of MS components. Among subjects presented less than four components of MS, the proportion of low HDL-C was the highest with the range of $44.73 \%$ to $68.79 \%$, and the proportion of elevated TG was the lowest with the range of $5.85 \%$ to $45.84 \%$. Among subjects presented four components of MS, the proportion of elevated BP was the highest (86.14\%).

\section{All-cause mortality rates}

There were 1,749 persons died during the median 7.44-years follow-up, the mortality rate was 46 per 10,000 person years. 
The mortality rates of MS individual components was shown in Table 3. Subjects with MS had a significantly higher mortality rate than Non-MS Subjects (71 vs. 35 per 10,000 personyears). In comparison to subjects without individual MS component, subjects with individual MS component significantly increased the risk of all-cause mortality except for HDL-C component.

\section{Relationships between all-cause mortality and MS}

Log-Rank test was used to determine proportional hazard assumption. For MS status, $P=0.1362>0.05$; for different number of MS components, $P=0.5294>0.05$. The KaplanMeier survival curves were shown in Fig 2.

Subjects with MS significantly increased the risk of all-cause mortality (Model 1 in Table 4, HR: 2.02, 95\% CI: 1.84-2.22). This increased risk remained significantly after adjustments for age, sex and classical risk factors (Model 3 in Table 4, HR: 1.26, 95\% CI: 1.14-1.40). Subjects with elevated BP, elevated FPG and central OB also significantly increased the risk of all-cause mortality by 1.34 (95\%CI: 1.20-1.50), 1.33 (95\%CI: 1.21-1.47) and 1.12(95\%CI: 1.01-1.25), respectively (Model 3 in Table 4). No significant statistically differences were found between all-cause mortality versus low HDL-C (Model 1,2,3 in Table 4) and elevated TG (Model 3 in Table 4).

Table 5 showed the relationships between 32 kinds of MS specific components combinations and all-cause mortality. HRs of different number of MS components decreased after adjusted for age and sex (Model 2 in Table 5). This decreased risk remained significantly after adjusted for age, sex and classical risk factors (Model 3 in Table 5). Otherwise, compared with 0 MS component, HRs increased significantly with the increasing number of MS components, especially when the number of MS components more than three. The HRs for those with 0 to 5 components were $1,1.22,1.25,1.33,1.66,1.92$, respectively (Model 3 in Table 5). There was a significant dose-response relationship $(P$ for liner trend $<0.001)$ between the number of MS components and the risk of all-cause mortality in the overall fixed cohort sample.

After adjusted for age, sex and classical risk factors, HRs of 32 kinds of MS specific components combinations decreased except for the individual MS component HDL-C. The all-cause mortality were more highly significant than other combinations $(P<0.05)$ when the following combinations exist: “elevated BP (HR: 1.36, 95\%CI: 1.06-1.73)”, “elevated FPG + low HDL-C (HR: 1.51, 95\%CI: 1.06-2.15)”, “elevated BP + elevated TG + elevated FPG (HR: 1.72, 95\%CI: 1.18-2.51)", "elevated FPG+ low HDL-C + elevated BP + elevated TG (HR: 2.05, 95\%CI: 1.50$2.80)$ ". None of these highest groups included central OB. (Table 5 and Fig B in S2 File)

\section{Relationships between all-cause mortality and MS after excluded baseline subjects with $\mathrm{EH}, \mathrm{T} 2 \mathrm{DM}$ and CKD}

After excluded baseline subjects with EH, T2DM and CKD, cox proportional hazards regression models were used to obtain HRs and 95\%CIs for all-cause mortality for MS individual

Table 2. Distribution of the study subjects among different number of MS components.

\begin{tabular}{|c|c|c|c|c|c|c|}
\hline Number of MS components & $N(45542)$ & Central OB & elevated BP & elevated TG & Low HDL-C & elevated FPG \\
\hline 0 & 7793 & 0 & 0 & 0 & 0 & 0 \\
\hline 1 & 12608 & 10.17 & 24.36 & 5.85 & 44.73 & 14.9 \\
\hline 2 & 11162 & 34.5 & 49.57 & 23.69 & 55.92 & 36.32 \\
\hline 3 & 7821 & 61.76 & 68.18 & 45.84 & 68.79 & 55.44 \\
\hline 4 & 4583 & 82.68 & 86.14 & 72.77 & 83.92 & 74.49 \\
\hline 5 & 1575 & 100 & 100 & 100 & 100 & 100 \\
\hline
\end{tabular}

OB: obesity; BP: diastolic blood pressure; TG: Triglyceride; HDL-C: high-density lipoprotein cholesterol; FPG: fasting plasma glucose.

doi:10.1371/journal.pone.0154990.t002 
Table 3. Mortality rate of MS individual components.

\begin{tabular}{|c|c|c|c|c|}
\hline MS components & $\mathbf{N}$ & Pearson-year & Died & Mortality rate $*(95 \% \mathrm{Cl})$ \\
\hline \multicolumn{5}{|l|}{ Central OB } \\
\hline No & 30215 & 251847 & 960 & $38(36-41)$ \\
\hline Yes & 15327 & 126829 & 789 & $62(58-67)$ \\
\hline \multicolumn{5}{|l|}{ elevated BP } \\
\hline No & 26083 & 218484 & 585 & $27(25-29)$ \\
\hline Yes & 19459 & 160192 & 1164 & 73(69-77) \\
\hline \multicolumn{5}{|l|}{ elevated TG } \\
\hline No & 33665 & 280330 & 1177 & $42(40-44)$ \\
\hline Yes & 11877 & 98346 & 572 & $58(54-63)$ \\
\hline \multicolumn{5}{|l|}{ Low HDL-C } \\
\hline No & 22860 & 190127 & 872 & $46(43-49)$ \\
\hline Yes & 22682 & 188549 & 877 & $47(44-50)$ \\
\hline \multicolumn{5}{|l|}{ Elevated FPG } \\
\hline No & 30285 & 252663 & 890 & $35(33-38)$ \\
\hline Yes & 15257 & 126013 & 859 & $68(64-73)$ \\
\hline \multicolumn{5}{|l|}{ MS-ATPIII } \\
\hline No & 31563 & 190127 & 929 & $35(33-38)$ \\
\hline Yes & 13979 & 188549 & 820 & $71(66-76)$ \\
\hline Total & 45542 & 378676 & 1749 & $46(44-48)$ \\
\hline
\end{tabular}

OB: obesity; BP: diastolic blood pressure; TG: Triglyceride; HDL-C: high-density lipoprotein cholesterol; FPG: fasting plasma glucose.

*: The unit of mortality rate was per 10,000 person-years.

doi:10.1371/journal.pone.0154990.t003

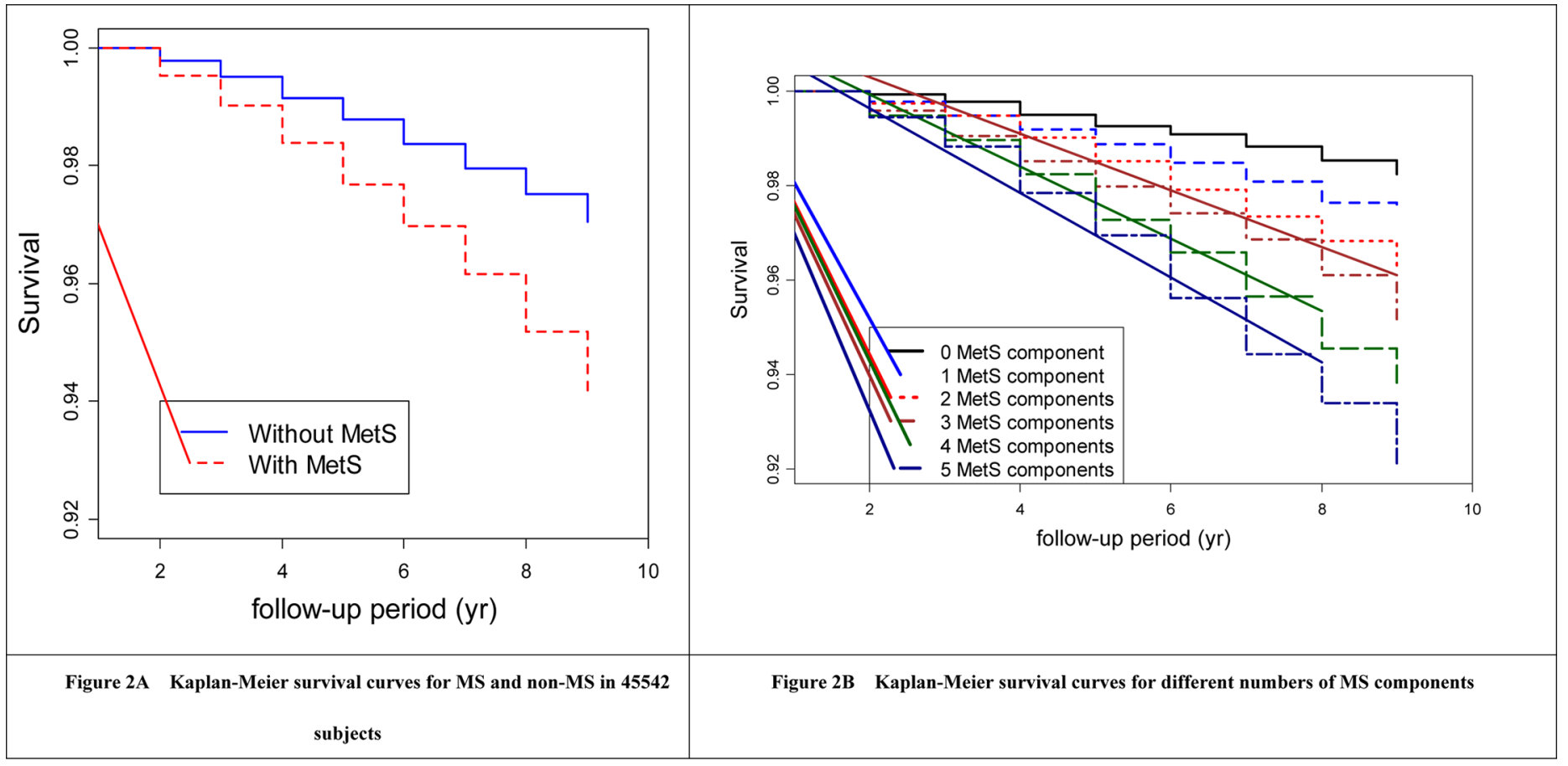

Fig 2. Kaplan-Meier survival curves for subjects.

doi:10.1371/journal.pone.0154990.g002 
Table 4. All-cause mortality associated with MS and its individual components.

\begin{tabular}{|c|c|c|c|c|}
\hline \multirow[t]{2}{*}{ MS components } & \multirow[t]{2}{*}{ Died } & \multicolumn{3}{|c|}{$\mathrm{HR}(95 \% \mathrm{Cl})$} \\
\hline & & Model 1 & Model 2 & Model 3 \\
\hline \multicolumn{5}{|l|}{ Central OB } \\
\hline No & 960 & - & - & - \\
\hline Yes & 789 & $1.64(1.49-1.80)^{* *}$ & $1.16(1.05-1.28)^{* *}$ & $1.12(1.01-1.25)^{*}$ \\
\hline \multicolumn{5}{|l|}{ elevated BP } \\
\hline No & 585 & - & - & - \\
\hline Yes & 1164 & $2.73(2.47-3.01)^{* *}$ & $1.36(1.22-1.51)^{* *}$ & $1.34(1.20-1.50)^{* *}$ \\
\hline \multicolumn{5}{|l|}{ elevated TG } \\
\hline No & 1177 & - & - & - \\
\hline Yes & 572 & $1.39(1.26-1.53)^{* *}$ & $1.16(1.05-1.28)^{* *}$ & $1.11(0.99-1.23)$ \\
\hline \multicolumn{5}{|l|}{ Low HDL-C } \\
\hline No & 872 & - & - & - \\
\hline Yes & 877 & $1.02(0.92-1.12)$ & $1.11(1.00-1.22)$ & $1.07(0.97-1.18)$ \\
\hline \multicolumn{5}{|l|}{ elevated FPG } \\
\hline No & 890 & - & - & - \\
\hline Yes & 859 & $1.94(1.77-2.13)^{* *}$ & $1.30(1.18-1.43)^{* *}$ & $1.33(1.21-1.47)^{* *}$ \\
\hline \multicolumn{5}{|l|}{ MS-ATPIII ${ }^{\wedge}$} \\
\hline No & 929 & - & - & - \\
\hline Yes & 820 & $2.02(1.84-2.22)^{* *}$ & $1.29(1.17-1.42)^{* *}$ & $1.26(1.14-1.40)^{* *}$ \\
\hline
\end{tabular}

HR: hazard ratios

*: $P<0.05$

**: $P<0.01$

A : compared with subjects presented less than three components of MS

Model 1: Unadjusted hazard ratios $(95 \% \mathrm{Cl})$

Model 2: Hazard ratios $(95 \% \mathrm{Cl})$ adjusted for age, sex

Model 3: Hazard ratios $(95 \% \mathrm{Cl})$ adjusted for age, sex, education, smoking, alcohol drinking, physical activity, EH family history and T2DM family history.

doi:10.1371/journal.pone.0154990.t004

components. Before adjustment, central OB, elevated FPG and elevated BP were associated with a significantly elevated risk of all-cause mortality (Model 1, Table D in S1 File). The HRs of central OB (1.54, 95\%CI: 1.28-1.85) and elevated FPG (1.40, 95\%CI: 1.16-1.68) were even higher than that of subjects with MS (1.38, 95\%CI: 1.12-1.71)). After adjusted for age and sex, there was no statistically significant differences between MS individual components and allcause mortality. (Model 2, Table D in S1 File)

Model 1 in Table 6 showed that the HRs increased with the number of MS components increasing from 2 to 4 before adjustment. When adjusted for age and sex, there showed no statistically significant differences between different number of MS components and all-cause mortality (Model 2 in Table 6).

After adjusted for age and sex, HRs of 32 kinds of MS specific components combinations decreased except for the individual MS component HDL-C. Besides, there showed no statistically significant differences between 32 kinds of MS specific components combinations and allcause mortality except for individual MS component central OB (1.69, 95\%CI: 1.17-2.45) (Model 2, Table E in S1 File)

After adjusted for MS individual components each other, elevated BP, elevated FPG and central OB increased the risk of all-cause mortality by 2.36 (95\%CI: $2.13-2.62$ ), 1.56 (95\%CI: $1.42-1.72)$ and 1.22 (95\%CI:1.10-1.34), respectively. No statistically significant differences 
Table 5. All-cause mortality associated with 32 kinds of MS specific component combinations.

\begin{tabular}{|c|c|c|c|c|c|}
\hline \multirow[t]{2}{*}{32 kinds of MS specific component combinations } & \multirow[t]{2}{*}{$\mathbf{N}$} & \multirow[t]{2}{*}{ Died } & \multicolumn{3}{|c|}{$\mathrm{HR}(95 \% \mathrm{Cl})$} \\
\hline & & & Model 1 & Model 2 & Model 3 \\
\hline 0 & 7793 & 136 & reference & reference & reference \\
\hline $1 \S$ & 12608 & 355 & $1.63(1.33-1.98)^{* *}$ & $1.25(1.03-1.53)^{*}$ & $1.22(0.99-1.49)$ \\
\hline OB & 1282 & 47 & $2.12(1.52-2.95)^{* *}$ & $1.47(1.06-2.05)^{*}$ & $1.40(0.99-2.00)$ \\
\hline $\mathrm{BP}$ & 3071 & 150 & $2.86(2.27-3.60)^{* *}$ & $1.40(1.11-1.77)^{* *}$ & $1.36(1.06-1.73)^{*}$ \\
\hline TG & 738 & 12 & $0.94(0.52-1.69)$ & $0.80(0.44-1.43)$ & $0.61(0.31-1.19)$ \\
\hline HDL-C & 5639 & 85 & $0.86(0.66-1.13)$ & $1.08(0.83-1.42)$ & $1.06(0.80-1.40)$ \\
\hline FPG & 1878 & 61 & $1.88(1.39-2.54)^{* *}$ & $1.26(0.93-1.70)$ & $1.31(0.96-1.79)$ \\
\hline $2 \S$ & 11162 & 438 & $2.28(1.88-2.76)^{* *}$ & $1.31(1.08-1.59)^{* *}$ & $1.25(1.02-1.53)^{*}$ \\
\hline $\mathrm{OB}, \mathrm{BP}$ & 1392 & 77 & $3.24(2.45-4.29)^{* *}$ & $1.42(1.07-1.88)^{*}$ & $1.36(1.01-1.83)^{*}$ \\
\hline $\mathrm{OB}, \mathrm{TG}$ & 317 & 6 & $1.09(0.48-2.47)$ & $0.79(0.35-1.79)$ & $0.64(0.26-1.57)$ \\
\hline OB,HDL-C & 1552 & 28 & $1.03(0.69-1.55)$ & $0.91(0.61-1.37)$ & $0.89(0.58-1.36)$ \\
\hline OB,FPG & 590 & 20 & $1.95(1.22-3.12)^{* *}$ & $1.10(0.69-1.76)$ & $1.07(0.64-1.77)$ \\
\hline $\mathrm{BP}, \mathrm{TG}$ & 592 & 30 & $2.97(200-4.41)^{* *}$ & $1.47(0.99-2.18)$ & $1.39(0.92-2.10)$ \\
\hline BP,HDL-C & 1878 & 88 & $2.74(2.10-3.59)^{* *}$ & $1.49(1.14-1.95)^{* *}$ & $1.31(0.98-1.75)$ \\
\hline $\mathrm{BP}, \mathrm{FPG}$ & 1671 & 108 & $3.81(2.96-4.90)^{* *}$ & $1.45(1.12-1.87)^{* *}$ & $1.48(1.14-1.93)^{* *}$ \\
\hline TG,HDL-C & 1377 & 25 & $1.04(0.68-1.59)$ & $0.98(0.64-1.49)$ & $0.90(0.58-1.40)$ \\
\hline TG,FPG & 358 & 11 & $1.78(0.96-3.29)$ & $1.17(0.63-2.16)$ & $1.13(0.59-2.15)$ \\
\hline HDL-C,FPG & 1435 & 45 & $1.81(1.29-2.54)^{* *}$ & $1.51(1.08-2.11)^{*}$ & $1.51(1.06-2.15)^{*}$ \\
\hline $3 \S$ & 7821 & 382 & $2.85(2.34-3.47)^{* *}$ & $1.39(1.14-1.7)^{* *}$ & $1.33(1.08-1.64)^{* *}$ \\
\hline $\mathrm{OB}, \mathrm{BP}, \mathrm{TG}$ & 533 & 25 & $2.75(1.80-4.22)^{* *}$ & $1.24(0.81-1.91)$ & $1.16(0.73-1.82)$ \\
\hline OB,BP,HDL-C & 1348 & 66 & $2.87(2.14-3.85)^{* *}$ & $1.34(0.99-1.80)$ & $1.24(0.90-1.70)$ \\
\hline $\mathrm{OB}, \mathrm{BP}, \mathrm{FPG}$ & 1139 & 88 & $4.54(3.47-5.94)^{* *}$ & $1.65(1.25-2.17)^{* *}$ & $1.65(1.24-2.20)^{* *}$ \\
\hline $\mathrm{OB}, \mathrm{TG}, \mathrm{HDL}-\mathrm{C}$ & 785 & 21 & $1.54(0.97-2.44)$ & $1.20(0.76-1.90)$ & $1.07(0.66-1.73)$ \\
\hline $\mathrm{OB}, \mathrm{TG}, \mathrm{FPG}$ & 277 & 10 & $2.08(1.10-3.96)^{*}$ & $1.20(0.63-2.28)$ & $1.20(0.61-2.36)$ \\
\hline OB,HDL-C,FPG & 748 & 28 & $2.16(1.44-3.25)^{* *}$ & $1.42(0.94-2.13)$ & $1.33(0.87-2.05)$ \\
\hline BP,TG,HDL-C & 819 & 32 & $2.28(1.55-3.35)^{* *}$ & $1.23(0.83-1.81)$ & $1.21(0.81-1.81)$ \\
\hline BP,HDL-C,FPG & 1001 & 58 & $3.42(2.51-4.65)^{* *}$ & $1.50(1.10-2.04)^{*}$ & $1.52(1.10-2.10)^{*}$ \\
\hline $\mathrm{BP}, \mathrm{TG}, \mathrm{FPG}$ & 492 & 37 & $4.43(3.08-6.37)^{* *}$ & $1.75(1.22-2.53)^{* *}$ & $1.72(1.18-2.51)^{* *}$ \\
\hline TG,HDL-C,FPG & 679 & 17 & $1.44(0.87-2.38)$ & $1.08(0.66-1.80)$ & $0.92(0.53-1.59)$ \\
\hline $4 \S$ & 4583 & 306 & $3.93(3.21-4.81)^{* *}$ & $1.76(1.43-2.16)^{* *}$ & $1.66(1.34-2.05)^{* *}$ \\
\hline OB,TG,HDL-C,BP & 1169 & 62 & $3.11(2.31-4.20)^{* *}$ & $1.51(1.12-2.05)^{* *}$ & $1.42(1.03-1.95)^{*}$ \\
\hline $\mathrm{OB}, \mathrm{TG}, \mathrm{BP}, \mathrm{FPG}$ & 737 & 54 & $4.32(3.15-5.92)^{* *}$ & $1.80(1.31-2.47)^{* *}$ & $1.67(1.20-2.34)^{* *}$ \\
\hline OB,HDL-C,BP,FPG & 1248 & 92 & $4.36(3.34-5.68)^{* *}$ & $1.80(1.37-2.35)^{* *}$ & $1.71(1.28-2.28)^{* *}$ \\
\hline OB,TG,HDL-C,FPG & 635 & 33 & $3.03(2.07-4.43)^{* *}$ & $1.87(1.27-2.73)^{* *}$ & $1.73(1.16-2.58)^{* *}$ \\
\hline TG,HDL-C,BP,FPG & 794 & 65 & $4.86(3.62-6.54)^{* *}$ & $2.08(1.54-2.80)^{* *}$ & $2.05(1.50-2.80)^{* *}$ \\
\hline $5 \S$ & 1575 & 132 & $4.97(3.92-6.32)^{* *}$ & $2.09(1.64-2.67)^{* *}$ & $1.92(1.48-2.49)^{* *}$ \\
\hline OB,HDL-C,FPG,BP,TG & 1575 & 132 & $4.97(3.91-6.32)^{* *}$ & $2.13(1.67-2.72)^{* *}$ & $1.97(1.52-2.55)^{* *}$ \\
\hline
\end{tabular}

HR: hazard ratios

$*: P<0.05$

**: $P<0.01$

$\S:$ compared with subjects present zero MS components

Model 1: Unadjusted hazard ratios $(95 \% \mathrm{Cl})$

Model 2: Hazard ratios $(95 \% \mathrm{Cl})$ adjusted for age, sex

Model 3: Hazard ratios $(95 \% \mathrm{Cl})$ adjusted for age, sex, education, smoking, alcohol drinking, physical activity, EH family history and T2DM family history.

doi:10.1371/journal.pone.0154990.t005 
Table 6. Relationships between different number of MS components and all-cause mortality after exclude subjects with EH or T2DM or CKD.

\begin{tabular}{|c|c|c|c|c|c|}
\hline \multirow[t]{2}{*}{ Number of MS components } & \multirow[t]{2}{*}{$\mathbf{N}$} & \multirow[t]{2}{*}{ Died } & \multicolumn{3}{|c|}{$\mathrm{HR}(95 \% \mathrm{Cl})$} \\
\hline & & & Model 1 & Model 2 & Model 3 \\
\hline 0 & 7151 & 106 & reference & reference & reference \\
\hline 1 & 9655 & 172 & $1.21(0.95-1.54)$ & $1.12(0.88-1.42)$ & $1.09(0.85-1.40)$ \\
\hline 2 & 6419 & 130 & $1.37(1.06-1.77)^{*}$ & $1.05(0.81-1.35)$ & $0.98(0.75-1.28)$ \\
\hline 3 & 3111 & 75 & $1.64(1.22-2.20)^{* *}$ & $1.12(0.83-1.50)$ & $1.11(0.81-1.51)$ \\
\hline 4 & 1100 & 27 & $1.67(1.10-2.55)^{*}$ & $1.07(0.70-1.63)$ & $1.11(0.72-1.72)$ \\
\hline 5 & 169 & 4 & $1.61(0.59-4.37)$ & $0.88(0.32-2.38)$ & $0.71(0.23-2.24)$ \\
\hline
\end{tabular}

HR: hazard ratios

$*$ : $P<0.05$

**: $P<0.01$

Model 1: Unadjusted hazard ratios $(95 \% \mathrm{Cl})$

Model 2: Hazard ratios $(95 \% \mathrm{Cl})$ adjusted for age, sex

Model 3: Hazard ratios $(95 \% \mathrm{Cl})$ adjusted for age, sex, education, smoking, alcohol drinking, physical activity, EH family history and T2DM family history.

doi:10.1371/journal.pone.0154990.t006

were found between all-cause mortality versus elevated TG or low HDL-C (Model 1 in Table 7). After further adjusted for age, sex and classical risk factors, statistically significant differences were only found between all-cause mortality versus elevated BP and elevated FPG (Model 3 in Table 7).

\section{Discussion}

In this retrospective cohort study we collected data related to age, sex, as well as demographic variables, and found that our data were distributed similarly to China's fifth census data. Besides, Yung-Hsuan's study showed that the data collected from MJ Health Screening Centers were distributed similarly to the general population in Taiwan [10]. However, subjects with MS accounted for $29.75 \%$ of the cohort (male: $30.36 \%$, female: $29.51 \%$ ), which is higher than has been found in Kuo-Chin's study (Taiwanese 22.4\%) [11] and Chien's study (male: 24.50\%; female: 19.90\%) [16]. This may be due to the use of different definitions of MS[17] and the difference in age distribution. The prevalence of MS increased significantly among subjects above 45 years old, and the prevalence of MS was higher in males compared with females, which was consistent with a relative study in China [18].

Table 7. Relationships between MS individual components and all-cause mortality after adjusted for each other.

\begin{tabular}{llll}
\hline & & \multicolumn{1}{c}{ HR(95\% CI) } & \\
\cline { 3 - 4 } & \multicolumn{1}{c}{ Model 1 } & \multicolumn{1}{c}{ Model 2 } & \multicolumn{1}{c}{ Model 3 } \\
\hline Central OB & $1.22(1.10-1.34)^{* *}$ & $1.07(0.97-1.18)$ & $1.04(0.94-1.16)$ \\
elevated BP & $2.36(2.13-2.62)^{* *}$ & $1.30(1.17-1.44)^{* *}$ & $1.29(1.15-1.45)^{* *}$ \\
elevated TG & $1.06(0.96-1.18)$ & $1.06(0.95-1.17)$ & $1.02(0.91-1.14)$ \\
Low HDL-C & $0.95(0.86-1.04)$ & $1.07(0.97-1.18)$ & $1.04(0.94-1.16)$ \\
elevated FPG & $1.56(1.42-1.72)^{* *}$ & $1.24(1.12-1.36)^{* *}$ & $1.28(1.16-1.42)^{* *}$ \\
\hline
\end{tabular}

**: $\mathrm{P}<0.001$

Model 1: adjusted for the other four MS components except for itself

Model 2: Model 1+adjusted for age, sex

Model 3: Model 2+adjusted for age, sex, education, smoking, alcohol drinking, physical activity, EH history family and T2DM family history.

doi:10.1371/journal.pone.0154990.t007 
The baseline characteristics of MS and Non-MS subjects showed that the main risk factors of MS were similar to those reported by present studies [19-25]. Our study showed that, subjects with MS had significantly higher age (54.63 \pm 10.36 vs. $48.25 \pm 10.3)$, T2DM family history ratio (12.07\% vs. $10.79 \%)$, EH family history ratio (18.66\% vs. $16.21 \%)$, weight, BMI, WC, central OB (72.92\% vs. 16.26\%), WHR, body fat, pulse, SBP (138.21 \pm 21.65 vs. $118.67 \pm 18.86)$, DBP $(81.45 \pm 11.83$ vs. $73.38 \pm 10.61)$, EH ratio (57.54\% vs. 17.21$)$, T2DM ratio $(14.71 \%$ vs. $1.91 \%)$ and CKD ratio (25.77\% vs. $11.97 \%$ ); but significantly lower education level (17.00\% vs. $27.33 \%$ ), height, timing for working on the seat, frequency of drinking milk, SSB and eating fruit. They

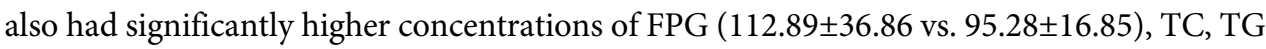
(178.61 \pm 91.95 vs. $100.53 \pm 50.59)$, LDL-C, CRP, ALT, AST, BUN, CRE and UA; but significantly lower HDL-C (39.88 \pm 11.66 vs. $49.16 \pm 13.35)$ and GFR. There also exist statistically significant differences on marriage status, frequency of physical activity, smoking and alcohol drinking between MS and Non-MS. No significant differences were found between the two groups on sex or cerebrovascular history $(\mathrm{P}>0.05)$.

In present cohort, more than $50 \%$ subjects had a low level of HDL-C. And among subjects presented less than four components of MS, the proportion of low HDL-C and elevated BP were the highest, and the proportion of elevated TG was the lowest.

In the present study, we studied the MS, MS individual components and 32 kinds of MS components combinations of all-cause mortality. There were 1,749 persons died during the median 7.44-years follow-up, the mortality rate was 46 per 10,000 person years. Subjects with MS had a significantly higher mortality rate than Non-MSNon-MS subjects (71 vs. 35 per 10,000 person-years), and subjects with individual MS components also significantly increased the risk of all-cause mortality except for HDL-C component.

This study demonstrated that Taiwanese with MS significantly increased the risk of all-cause mortality compared with subjects without MS, which was consistent with previous studies [2628]. Besides, subjects with elevated BP, elevated FPG, central OB and had a significantly higher rates of all-cause mortality. The increased risk remained significantly after adjustments for age, sex and classical risk factors, and the mortality rate of subjects with MS was lower than that of subjects with elevated BP or elevated FPG, but higher than subjects with central OB. While, no significant statistically differences were found between all-cause mortality versus low HDL-C and elevated TG after adjustment, which was consistent with the results from Huang's study in 2008 [11]. Otherwise, when subjects with EH, T2DM and CKD were excluded, no significant statistically differences were found between all-cause mortality versus MS and MS individual components after adjusted age and sex. The results above indicates that EH, T2DM and CKD may beimportant stages before all-cause mortality, and the risk of all-cause mortality would not be increased once EH, T2DM and CKD were controlled, which was also demonstrated by previous studies[29-32]. Besides, Hsu's study[16] showed that when exclude subjects with EH and T2DM, compared with Non-MS, HRs of MS for males and females were 0.81(95\%CI: 0.51-1.30) and 1.14 (95\%CI: 0.45-2.92), respectively. Wang's [33] study also reported that no significant statistically differences were found between MS and all-cause mortality after excluded subjects with T2DM.

Although HRs for different number of MS components decreased after adjusted for age, sex and classical risk factors, there still existed a significant dose-response relationship ( $P$ for liner trend $<0.001$ )between the number of MS components and the risk of all-cause mortality. The HRs for subjects with 0 to 5 components were $1,1.22,1.25,1.33,1.66$, and 1.92, respectively. Similar results was also found in Trevisan's study[34], which included 20000 subjects and followed up for 7 years, the HRs for males with 0 to 5 components were $1,1.2,1.4,1.8,2$, respectively; the HRs for females with 0 to 5 components were $1,1.2,1.6,2.1,2.5$, respectively. However, after excluded subjects with EH, T2DM and CKD, no significant statistically differences were found between different number of MS components and all-cause mortality. 
Our study also identified several MS specific component combinations that posed the highest risk of developing all-cause mortality. As MS will continue to be an important burden to the population, identifying high-risk combinations of MS may help identify intervention strategies and cost-effectively utilize limited resources to reduce the burden of MS-related mortality in general. After adjusted for age, sex and classical risk factors, the all-cause mortality were more highly significant than other combinations $(P<0.05)$ when the following combinations exist: "elevated BP", "elevated FPG + low HDL-C", "elevated BP + elevated TG + elevated FPG", "elevated FPG+ low HDL-C + elevated BP + elevated TG". Similar results were indicated in relevant studies. Hong's [35] study reported that the following clusters of MS components posed the highest risk for all-cause mortality: "elevated BP + elevated FPG + Low HDL-C / elevated TG". Guize's[36] study also indicated that the combinations of "Central OB + elevated FPG + elevated BP / elevated TG" had the highest all-cause mortality rate. Otherwise, after excluded subjects with EH, T2DM and CKD, no significant statistically differences was found between any kinds of MS specific component combinations and all-cause mortality.

Our study has several limitations. First, there exists significantly ethnic differences among subjects with MS [37], which might have caused the non-statistically significant differences results. Second, the follow-up time was relatively short and therefore mortality endpoints may be caused by EH,T2DM and CKD. Third, the distribution of MS individual components varied a lot between different study population [38].

In conclusion, this cohort study of Taiwanese adults has shown that MS increased all-cause mortality before excluded subjects with EH,T2DM and CKD, which indicated that MS was an independent predictor of all-cause mortality. Because of the short follow-up time, non-significant higher risk of all-cause mortality was seen in subjects with MS, or MS individual components, or different kinds of MS components combinations after excluded subjects with $\mathrm{EH}$, T2DM and CKD, which indicated that the mortality endpoints were caused by EH,T2DM and CKD. Besides, there existed a significant dose-response relationship between the number of MS components and the risk of all-cause mortality, and this relationship disappeared after excluded subjects with EH,T2DM and CKD. Among different kinds of MS components combinations, the groups of "elevated BP +elevated FPG + elevated TG" and "elevated BP + elevated FPG + elevated TG +low HDL-C” posed the highest risk for all-cause mortality. Further research is needed to clarify whether the identification of MS individual components and different kinds of MS component combinations may help to distinguish subjects with higher risk of all-cause mortality. Subjects with MS, who had EH, T2DM and CKD at the same time, should pay more attention to their health status, be treated with lifestyle intervention, increase the frequency of taking health examinations, followed by drugs if necessary, seek immediate medical care once felt uncomfortable, especially for those who had the specific MS components combinations above.

\section{Supporting Information}

S1 File. Appendix Tables.

(PDF)

S2 File. Appendix Figures.

(PDF)

\section{Author Contributions}

Conceived and designed the experiments: FS SYZ. Performed the experiments: ZXL XHY JY ZRY SFW. Analyzed the data: ZXL XHY JY ZRY SFW. Contributed reagents/materials/ 
analysis tools: JY. Wrote the paper: ZXL XHY. Interpreting the results, draft reviewing, and finalizing the paper: FS SYZ.

\section{References}

1. Ford ES. Risks for all-cause mortality, cardiovascular disease, and diabetes associated with the metabolic syndrome: a summary of the evidence. Diabetes care. 2005; 28(7):1769-78. Epub 2005/06/29. PMID: 15983333.

2. Galassi A, Reynolds $\mathrm{K}, \mathrm{He} \mathrm{J}$. Metabolic syndrome and risk of cardiovascular disease: a meta-analysis. The American journal of medicine. 2006; 119(10):812-9. Epub 2006/09/27. doi: 10.1016/j.amjmed. 2006.02.031 PMID: 17000207.

3. Gami AS, Witt BJ, Howard DE, Erwin PJ, Gami LA, Somers VK, et al. Metabolic syndrome and risk of incident cardiovascular events and death: a systematic review and meta-analysis of longitudinal studies. Journal of the American College of Cardiology. 2007; 49(4):403-14. Epub 2007/01/30. doi: 10. 1016/j.jacc.2006.09.032 PMID: 17258085.

4. Zambon S, Zanoni S, Romanato G, Corti MC, Noale M, Sartori L, et al. Metabolic syndrome and allcause and cardiovascular mortality in an Italian elderly population: the Progetto Veneto Anziani (Pro.V. A.) Study. Diabetes care. 2009; 32(1):153-9. Epub 2008/10/22. doi: 10.2337/dc08-1256 PMID: $18931101 ;$ PubMed Central PMCID: PMCPmc2606852.

5. Sun DL, Wang JH, Jiang B, Li LS, Li LS, Wu L, et al. Metabolic syndrome vs. its components for prediction of cardiovascular mortality: A cohort study in Chinese elderly adults. Journal of geriatric cardiology: JGC. 2012; 9(2):123-9. Epub 2012/08/24. doi: 10.3724/sp.j.1263.2012.01172 PMID: 22916057; PubMed Central PMCID: PMCPmc3418900.

6. Chien KL, Hsu HC, Sung FC, Su TC, Chen MF, Lee YT. Metabolic syndrome as a risk factor for coronary heart disease and stroke: an 11-year prospective cohort in Taiwan community. Atherosclerosis. 2007; 194(1):214-21. Epub 2006/09/19. doi: 10.1016/j.atherosclerosis.2006.07.033 PMID: 16979176.

7. Ishizaka N, Ishizaka Y, Toda E, Hashimoto H, Nagai R, Yamakado M. Hypertension is the most common component of metabolic syndrome and the greatest contributor to carotid arteriosclerosis in apparently healthy Japanese individuals. Hypertension research: official journal of the Japanese Society of Hypertension. 2005; 28(1):27-34. Epub 2005/06/23. doi: 10.1291/hypres.28.27 PMID: 15969252.

8. Ohsawa M, Fujioka T, Ogasawara K, Tanno K, Okamura T, Turin TC, et al. High risks of all-cause and cardiovascular deaths in apparently healthy middle-aged people with preserved glomerular filtration rate and albuminuria: A prospective cohort study. International journal of cardiology. 2013; 170(2):16772. Epub 2013/11/12. doi: 10.1016/j.ijcard.2013.10.076 PMID: 24211064.

9. Lakka HM, Laaksonen DE, Lakka TA, Niskanen LK, Kumpusalo E, Tuomilehto J, et al. The metabolic syndrome and total and cardiovascular disease mortality in middle-aged men. Jama. 2002; 288 (21):2709-16. Epub 2002/12/04. PMID: 12460094.

10. Shen YH, Yang WS, Lee TH, Lee LT, Chen CY, Huang KC. Bright liver and alanine aminotransferase are associated with metabolic syndrome in adults. Obesity research. 2005; 13(7):1238-45. Epub 2005/ 08/04. doi: 10.1038/oby.2005.147 PMID: 16076994.

11. Hsu PF, Chuang SY, Cheng HM, Tsai ST, Chou P, Chen $\mathrm{CH}$. Clinical significance of the metabolic syndrome in the absence of established hypertension and diabetes: A community-based study. Diabetes research and clinical practice. 2008; 79(3):461-7. Epub 2007/11/17. doi: 10.1016/j.diabres.2007.09. 021 PMID: 18006171.

12. Wen CP, Cheng TY, Tsai MK, Chang YC, Chan HT, Tsai SP, et al. All-cause mortality attributable to chronic kidney disease: a prospective cohort study based on 462293 adults in Taiwan. Lancet. 2008; 371(9631):2173-82. doi: 10.1016/S0140-6736(08)60952-6 PMID: 18586172.

13. Wen CP, Wai JP, Tsai MK, Yang YC, Cheng TY, Lee MC, et al. Minimum amount of physical activity for reduced mortality and extended life expectancy: a prospective cohort study. Lancet. 2011; 378 (9798):1244-53. Epub 2011/08/19. doi: 10.1016/s0140-6736(11)60749-6 PMID: 21846575.

14. Third Report of the National Cholesterol Education Program (NCEP) Expert Panel on Detection, Evaluation, and Treatment of High Blood Cholesterol in Adults (Adult Treatment Panel III) final report. Circulation. 2002; 106(25):3143-421. Epub 2002/12/18. PMID: 12485966.

15. National Bureau of Statistics of the People's Republic of China. China's fifth census in 2000. Available: http://wwwstatsgovcn/tjsj/ndsj/renkoupucha/2000pucha/puchahtm. 2000.

16. Chien KL, Lee BC, Hsu HC, Lin HJ, Chen MF, Lee YT. Prevalence, agreement and classification of various metabolic syndrome criteria among ethnic Chinese: a report on the hospital-based health diagnosis of the adult population. Atherosclerosis. 2008; 196(2):764-71. Epub 2007/02/13. doi: 10.1016/j. atherosclerosis.2007.01.006 PMID: 17291512. 
17. Alberti KG, Eckel RH, Grundy SM, Zimmet PZ, Cleeman JI, Donato KA, et al. Harmonizing the metabolic syndrome: a joint interim statement of the International Diabetes Federation Task Force on Epidemiology and Prevention; National Heart, Lung, and Blood Institute; American Heart Association; World Heart Federation; International Atherosclerosis Society; and International Association for the Study of Obesity. Circulation. 2009; 120(16):1640-5. Epub 2009/10/07. doi: 10.1161/circulationaha.109.192644 PMID: 19805654.

18. Li Y, Zhao D, Wang W, Wang WH, Sun JY, Qin LP, et al. A comparison of three diagnostic criterions for metabolic syndrome applied in a Chinese population aged 35-64 in 11 provinces. Chinese Journal of Epidemiology. 2007; 28:83-7. PMID: 17575940

19. Stoutenberg M, Lee DC, Sui X, Hooker S, Horigian V, Perrino T, et al. Prospective study of alcohol consumption and the incidence of the metabolic syndrome in US men. The British journal of nutrition. 2013; 110(5):901-10. Epub 2013/01/24. doi: 10.1017/s0007114512005764 PMID: 23340203.

20. Broekhuizen LN, Boekholdt SM, Arsenault BJ, Despres JP, Stroes ES, Kastelein JJ, et al. Physical activity, metabolic syndrome, and coronary risk: the EPIC-Norfolk prospective population study. European journal of cardiovascular prevention and rehabilitation: official journal of the European Society of Cardiology, Working Groups on Epidemiology \& Prevention and Cardiac Rehabilitation and Exercise Physiology. 2011; 18(2):209-17. Epub 2011/04/01. doi: 10.1177/1741826710389397 PMID: 21450666.

21. Niwa $Y$, Ishikawa $S$, Gotoh $T$, Kayaba K, Nakamura $Y$, Kajii E. Association between stroke and metabolic syndrome in a Japanese population: Jichi Medical School (JMS) Cohort Study. Journal of epidemiology / Japan Epidemiological Association. 2010; 20(1):62-9. Epub 2009/12/08. PMID: 19966508; PubMed Central PMCID: PMCPmc3900781.

22. Sicras-Mainar A, Maurino J, Ruiz-Beato E, Navarro-Artieda R. Prevalence of metabolic syndrome according to the presence of negative symptoms in patients with schizophrenia. Neuropsychiatric disease and treatment. 2015; 11:51-7. Epub 2015/01/08. doi: 10.2147/ndt.s75449 PMID: 25565850; PubMed Central PMCID: PMCPmc4283985.

23. Harrison SL, Stephan BC, Siervo M, Granic A, Davies K, Wesnes KA, et al. Is there an association between metabolic syndrome and cognitive function in very old adults? The newcastle $85+$ study. Journal of the American Geriatrics Society. 2015; 63(4):667-75. Epub 2015/04/09. doi: 10.1111/jgs.13358 PMID: 25850570.

24. Choi KM, Cho HJ, Choi HY, Yang SJ, Yoo HJ, Seo JA, et al. Higher mortality in metabolically obese normal-weight people than in metabolically healthy obese subjects in elderly Koreans. Clinical endocrinology. 2013; 79(3):364-70. Epub 2013/01/22. doi: 10.1111/cen.12154 PMID: 23330616.

25. Larsen SB, Kroman N, Ibfelt EH, Christensen J, Tjonneland A, Dalton SO. Influence of metabolic indicators, smoking, alcohol and socioeconomic position on mortality after breast cancer. Acta oncologica (Stockholm, Sweden). 2015; 54(5):780-8. Epub 2015/03/12. doi: 10.3109/0284186x.2014.998774 PMID: 25761087.

26. Kondo T, Osugi S, Shimokata K, Honjo H, Morita Y, Yamashita K, et al. Metabolic syndrome and allcause mortality, cardiac events, and cardiovascular events: a follow-up study in 25,471 young- and middle-aged Japanese men. European journal of cardiovascular prevention and rehabilitation: official journal of the European Society of Cardiology, Working Groups on Epidemiology \& Prevention and Cardiac Rehabilitation and Exercise Physiology. 2011; 18(4):574-80. Epub 2011/04/01. doi: 10.1177/ 1741826710389529 PMID: 21450628.

27. Ao H, Xu F, Wang X, Tang X, Zheng Z, Hu S. Effects of metabolic syndrome with or without obesity on outcomes after coronary artery bypass graft. A cohort and 5-year study. PloS one. 2015; 10(2): e0117671. Epub 2015/02/14. doi: 10.1371/journal.pone.0117671 PMID: 25679397; PubMed Central PMCID: PMCPmc4332488.

28. van Bunderen CC, Oosterwerff MM, van Schoor NM, Deeg DJ, Lips P, Drent ML. Serum IGF1, metabolic syndrome, and incident cardiovascular disease in older people: a population-based study. European journal of endocrinology / European Federation of Endocrine Societies. 2013; 168(3):393-401. Epub 2012/12/13. doi: 10.1530/eje-12-0784 PMID: 23233113.

29. Stern MP, Williams K, Hunt KJ. Impact of diabetes/metabolic syndrome in patients with established cardiovascular disease. Atherosclerosis Supplements. 2005; 6(2):3-6. Epub 2005/04/13. doi: 10.1016/j. atherosclerosissup.2005.02.002 PMID: 15823490.

30. Howard BV, Lee ET, Cowan LD, Devereux RB, Galloway JM, Go OT, et al. Rising tide of cardiovascular disease in American Indians. The Strong Heart Study. Circulation. 1999; 99(18):2389-95. Epub 1999/ 05/11. PMID: 10318659.

31. Resnick HE, Jones K, Ruotolo G, Jain AK, Henderson J, Lu W, et al. Insulin resistance, the metabolic syndrome, and risk of incident cardiovascular disease in nondiabetic american indians: the Strong Heart Study. Diabetes care. 2003; 26(3):861-7. Epub 2003/03/01. PMID: 12610050. 
32. Zhang J, Liu NJ, Zhang $\mathrm{Y}$, Yang $\mathrm{H}$, Tang Z. The association and interaction analysis of metabolic syndrome and chronic kidney disease on cardiovascular autonomic neuropathy in the general Chinese population. International journal of clinical and experimental medicine. 2015; 8(6):9649-57. Epub 2015/ 08/27. PMID: 26309639; PubMed Central PMCID: PMCPmc4538176.

33. Wang J, Ruotsalainen S, Moilanen L, Lepisto P, Laakso M, Kuusisto J. The metabolic syndrome predicts cardiovascular mortality: a 13-year follow-up study in elderly non-diabetic Finns. European heart journal. 2007; 28(7):857-64. Epub 2007/02/17. doi: 10.1093/eurheartj/ehl524 PMID: 17303589.

34. Trevisan M, Liu J, Bahsas FB, Menotti A. Syndrome X and mortality: a population-based study. Risk Factor and Life Expectancy Research Group. American journal of epidemiology. 1998; 148(10):95866. Epub 1998/11/26. PMID: 9829867.

35. Hong Y, Jin X, Mo J, Lin HM, Duan Y, Pu M, et al. Metabolic syndrome, its preeminent clusters, incident coronary heart disease and all-cause mortality-results of prospective analysis for the Atherosclerosis Risk in Communities study. Journal of internal medicine. 2007; 262(1):113-22. Epub 2007/06/30. doi: 10.1111/j.1365-2796.2007.01781.x PMID: 17598819.

36. Guize L, Thomas F, Pannier B, Bean K, Jego B, Benetos A. All-cause mortality associated with specific combinations of the metabolic syndrome according to recent definitions. Diabetes care. 2007; 30 (9):2381-7. Epub 2007/06/15. doi: 10.2337/dc07-0186 PMID: 17563336.

37. Chuang SY CC, Chou P. Hypertension and the Insulin-related Metabolic Syndrome: Factor Analysis in 17,539 Taiwanese. Acta Cardiol Sin. 2004; 20:229-36.

38. Sheu WH, Chuang SY, Lee WJ, Tsai ST, Chou P, Chen CH. Predictors of incident diabetes, metabolic syndrome in middle-aged adults: a 10-year follow-up study from Kinmen, Taiwan. Diabetes research and clinical practice. 2006; 74(2):162-8. Epub 2006/04/20. doi: 10.1016/j.diabres.2006.03.011 PMID: 16621112. 